\title{
Optical limitations guide behavioral algorithms in Drosophila
}

\author{
Jessica L. Fox * \\ Department of Biology, Case Western Reserve University, Cleveland, OH, USA \\ ${ }^{*}$ Correspondence: jlf88@case.edu
}

Edited by:

Martin Giurfa, Université Paul Sabatier-Toulouse III, France

Reviewed by:

Martin Giurfa, Université Paul Sabatier-Toulouse III, France

Martin Gopfert, University of Gottingen, Germany

Keywords: Drosophila, saccades, optic flow, visual acuity, halteres, walking, head body coordination

\section{A commentary on}

Saccadic body turns in walking Drosophila

by Geurten, B. R. H., Jähde, $P$., Corthals, K., and Göpfert, M. C. (2014). Front. Behav. Neurosci. 8:365. doi: 10.3389/fnbeh.2014.00365

Vision is complicated by movement. As a seeing animal moves through space, its own movement causes the entire visual scene to move on the retina, creating a wide-field motion stimulus that may be irrelevant and confounds the visual system's ability to form a stable image. In both vertebrates and invertebrates, self-motion of the body is detected by mechanosensors (in vertebrates, the inner ear; in invertebrates, statoliths, or other organs), and countered by compensatory rotations of the eye that steady the image on the retina. These counter-rotations remove the irrelevant stimulus and allow the animal to focus on motion in the external environment. But what happens when the animal intentionally shifts its gaze to another part of its visual world?

In voluntary eye movements to fixate objects of interest, the eye is moved as fast as possible to minimize the time during which the visual image is blurred. These rapid movements are known as saccades, and they occur in human eyes so quickly that we don't notice them as we move our eyes across a page of text or a visual scene. Insects perform the same kind of fast fixating saccades, but since their eyes are fixed in their heads, their saccades take the form of full body rotations, which change the direction of movement. These body saccades serve multiple purposes. They minimize the time of motion blur as they do in vertebrates, and by minimizing the time spent in rotation they allow the fly to rapidly change heading. Because many insects lack stereoscopic vision for depth perception (Geurten et al., 2014), self-motion is also essential to obtaining depth information. Since translatory motions provide more distance cues than rotational motions, separating body movements into long periods of pure translation, interspersed with fast periods of pure rotation, is an effective method for organizing visuallyguided self-motion. Previous studies have found that fruit flies use this strategy when flying (Wolf and Heisenberg, 1980). However, when walking, their forward speed is lower, and the demands on visual control systems are quite different from those in flight. Do flies also follow this motion-vision strategy while they walk?

A recent paper by Geurten et al. (2014) indicates that the algorithm for organizing visually-guided flight is also followed on the ground. Using videos of walking flies and k-means clustering, Geurten et al. sorted fly walking behaviors into several prototypical movements (PMs). They find that walking flies, like flying flies, have a small number of distinct PMs, with different amounts of time spent in each. The flies show longer periods of translatory (straight) movement, interspersed with short times spent in rotational saccades (as well as rest periods-which is how the flies spend the majority of their time). Walking saccades are slower than those during flight, but are similar in duration and amplitude to those observed in walking blowflies (Calliphora), and bees (Apis). Thus, at first glance, Drosophila walking behavior appears to follow the same saccade algorithms seen in other walking insects (Blaj and van Hateren, 2004).

However, there are two means by which an insect can make a saccade, with very distinct sensorimotor consequences for each: the insect can rotate its entire body and change its heading, or it can use its highly flexible neck to simply rotate its head. In bees and blowflies, both kinds of saccades are observed, but Geurten et al. observed that the rotational saccades of fruit flies are not accompanied by independent head saccades. Why not?

The answer may lie in the optics of the eyes. The spatial and temporal resolution of the eyes of Drosophila is significantly lower than that of the eyes of Apis or Calliphora (Laughlin and Horridge, 1971; Petrowitz et al., 2000). Geurten et al. modeled the effects of a head saccade on the visual scene by processing several different images with a filter based on the measured optics of the fly's eye, to gain an understanding of what the fly sees. They found that Drosophila would have to move its head well beyond the physically possible range to obtain an image shift comparable to those observed from Calliphora or Apis. Thus, Drosophila turns its entire body rather than its head.

How does this compare with fruit fly head movement behavior during flight? A satisfactory answer is not yet available. The heads of freely-flying fruit flies are difficult to resolve in video, so we have resorted to tethered flight as a substitute. In flying flies that cannot rotate their bodies, 
head saccades are prevalent, and the head closely follows moving wide-field visual stimuli to stabilize it on the retina (Fox and Frye, 2014). In other experiments, larger flies (Calliphora and Musca) were tethered to a pin such that they could freely rotate within a circular visual display (Land, 1973; Geiger and Poggio, 1977; Bender and Dickinson, 2006), or magnetic coils were used to observe head movements in free flight (Schilstra and van Hateren, 1998; van Hateren and Schilstra, 1999). Although there is some disagreement in those studies about whether flies move their heads independently of their bodies during flight, or whether the observations in larger flies are applicable to fruit flies, it is clear that all flies perform saccades with the entire body. These saccades are visually initiated, but their duration and magnitude is determined by feedback from the mechanosensory halteres (Bender and Dickinson, 2006), which are oscillated in flight and detect body rotations.

Geurten et al. note that Drosophila's lack of head rotations in walking is accompanied by a lack of haltere movements during walking. This is in sharp contrast to the walking behavior of Calliphora, which performs both head saccades (Blaj and van Hateren, 2004), and haltere oscillations (Sandeman and Markl, 1980) during walking behavior. Although this data is only correlative for now, it is an important observation that underscores the diversity of fly behaviors. This study suggests that there may be multiple strategies for gaze control during walking in dipteran insects: one in which a high-resolution visual system is rotated rapidly under the influence of the oscillating halteres, as in Calliphora, and another in which a lower-resolution system remains more or less fixed to the rotating body without haltere input. Their study raises important questions about both the distinction between these strategies, and how the overall circuitry of the fly's sensorimotor system might be adapted to particular sensory, contextual, and ecological constraints.

\section{REFERENCES}

Bender, J. A., and Dickinson, M. H. (2006). A comparison of visual and haltere-mediated feedback in the control of body saccades in Drosophila melanogaster. J. Exp. Biol. 209, 4597-4606. doi: $10.1242 /$ jeb. 02583

Blaj, G., and van Hateren, J. H. (2004). Saccadic head and thorax movements in freely walking blowflies. J. Comp. Phys. A 190, 861-868. doi: 10.1007/s00359-004-0541-4

Fox, J. L., and Frye, M. A. (2014). Figure-ground discrimination behavior in Drosophila. II. Visual influences on head movement behavior. J. Exp. Biol. 217, 570-579. doi: 10.1242/jeb.080192

Geiger, G., and Poggio, T. (1977). On head and body movements of flying flies. Biol. Cybern. 25, 177-180. doi: 10.1007/BF00365214

Geurten, B. R. H., Jähde, P., Corthals, K., and Göpfert, M. C. (2014). Saccadic body turns in walking Drosophila. Front. Behav. Neurosci. 8:365. doi: 10.3389/fnbeh.2014.00365

Land, M. F. (1973). Head movement of flies during visually guided flight. Nature 243, 299-300. doi: $10.1038 / 243299 \mathrm{a} 0$
Laughlin, S. B., and Horridge, G. A. (1971). Angular sensitivity of the retinula cells of dark-adapted worker bee. Zeitschrift für Vergleichende Physiologie 74, 329-335. doi: 10.1007/BF00297733

Petrowitz, R., Dahmen, H., Egelhaaf, M., and Krapp, H. G. (2000). Arrangement of optical axes and spatial resolution in the compound eye of the female blowfly Calliphora. J. Comp. Physiol. A 186, 737-746. doi: 10.1007/s003590000127

Sandeman, D. C., and Markl, H. (1980). Head movements in flies (Calliphora) produced by deflexion of the halteres. J. Exp. Biol. 85, 43-60.

Schilstra, C., and van Hateren, J. H. (1998). Stabilizing gaze in flying blowflies. Nature 395, 654-654. doi: $10.1038 / 27114$

van Hateren, J. H., and Schilstra, C. (1999). Blowfly flight and optic flow. II. Head movements during flight. J. Exp. Biol. 202(Pt 11), 1491-1500.

Wolf, R., and Heisenberg, M. (1980). On the fine structure of yaw torque in visual flight orientation of Drosophila melanogaster. J. Comp. Phys. A 140, 69-80. doi: 10.1007/BF00613749

Conflict of Interest Statement: The author declares that the research was conducted in the absence of any commercial or financial relationships that could be construed as a potential conflict of interest.

Received: 09 October 2014; accepted: 10 October 2014; published online: 31 October 2014.

Citation: Fox JL (2014) Optical limitations guide behavioral algorithms in Drosophila. Front. Behav. Neurosci. 8:374. doi: 10.3389/fnbeh.2014.00374

This article was submitted to the journal Frontiers in Behavioral Neuroscience.

Copyright (C) 2014 Fox. This is an open-access article distributed under the terms of the Creative Commons Attribution License (CC BY). The use, distribution or reproduction in other forums is permitted, provided the original author(s) or licensor are credited and that the original publication in this journal is cited, in accordance with accepted academic practice. No use, distribution or reproduction is permitted which does not comply with these terms. 